\title{
OPEN CENTRALIZERS AND THE CONTINUITY OF GROUP REPRESENTATIONS
}

\author{
CLAUDE SCHOCHET AND BERTRAM SCHREIBER ${ }^{1}$
}

\begin{abstract}
Let $G$ be a locally compact group, $\pi: G \rightarrow \mathfrak{L}\left(L^{2}(G)\right)$ the right regular representation of $G$, and $G^{c}=\left\{x \in G\right.$ : the function $g \leadsto \pi\left(g x g^{-1}\right)$ is norm continuous\}. This note is devoted to the study of $G^{c}$. In particular, the compactly generated groups for which $G=G^{c}$ are characterized.
\end{abstract}

1. Let $G$ be a locally compact group and let $\pi: G \rightarrow \mathfrak{L}\left(L^{2}(G)\right)$ be the right regular representation of $G$ on $L^{2}(G)$ with respect to a right Haar measure. The function $\pi$ is continuous when $\mathcal{L}\left(L^{2}(G)\right)$ is given the strong operator topology, but $\pi$ is not continuous with respect to the norm topology, except in trivial cases. Nevertheless, there is a middle ground, to which this note is devoted.

Following [5], [8], let $\mathfrak{L}_{G}=\left\{T \in \mathcal{L}\left(L^{2}(G)\right)\right.$ : the function $g \leadsto \pi(g) T \pi(g)^{*}$ is norm continuous $\}$. Then $\mathscr{L}_{G}$ is a $C^{*}$-algebra which contains the compact operators and has various pleasing properties (cf. [5, Theorem 2.2]). Let

$$
\begin{aligned}
G^{c} & =\left\{x \in G: \pi(x) \in \mathfrak{L}_{G}\right\} \\
& =\left\{x \in G: \text { the function } g \leadsto \pi\left(g x g^{-1}\right) \text { is norm continuous }\right\} .
\end{aligned}
$$

It is easy to see that $G^{c}$ is a subgroup of $G$. If $G$ is abelian or discrete, then $G^{c}=G$; in general it is much smaller. The relationship between $G^{c}$ and $G$ is the main subject of this note.

We shall denote the identity component and the center of $G$ by $G_{0}$ and $Z(G)$, respectively. For $x, y \in G, C_{G}(x)$ denotes the centralizer of $x$ in $G$ and $[x, y]=$ $x y x^{-1} y^{-1}$.

Definition 1.1. Let $B$ be a Banach space of functions on $G$. Suppose that there exist constants $C, \delta>0$ such that the following conditions are satisfied:

(i) If $\varphi \in B$ and $x \in G$, then ${ }_{B} \pi(x) \varphi \in B$, and $\left\|_{B} \pi(x) \varphi\right\|<C\|\varphi\|$, where ${ }_{B} \pi(x) \varphi(t)=\varphi\left(t x^{-1}\right)$.

(ii) Given $\varphi \in B$, there exists $\lambda(\varphi)>0$ such that $\|\varphi+\psi\|>\lambda(\varphi)$ for all $\psi \in B$ such that $\varphi \cdot \psi=0$.

(iii) For every neighborhood $U$ of $e$ in $G$ there exists $0 \neq \varphi \in B$ such that $\varphi=0$ off $U$ and $\lambda(\varphi)>\delta\|\varphi\|$.

Then $B$ will be called a homogeneous separating Banach space of functions on $G$.

Received by the editors March 13, 1980 and, in revised form, June 18, 1980.

1980 Mathematics Subject Classification. Primary 47C15, 47D10.

${ }^{1}$ Research of both authors was partially supported by the National Science Foundation. 
EXAMPLES 1.2. Most of the Banach spaces commonly encountered in harmonic analysis satisfy the conditions of Definition 1.1. The following is a sampling of such spaces.

(i) $L^{p}(G), 1 \leqslant p \leqslant \infty$,

(ii) $C_{0}(G)$,

(iii) the Fourier algebra $A(G)[1]$,

(iv) the Sobolev spaces $W_{k}^{p}(G)$ for $G$ a Lie group,

(v) the spaces $A_{p}^{q}(G)=L^{p}(G) \hat{\otimes} L^{q}(G)$ of Figà-Talamanca and Gaudry [2], [3] on $G \times G$.

Definition 1.3. Let $B$ be a homogeneous separating Banach space of functions on $G$ and ${ }_{B} \pi: G \rightarrow \mathcal{L}(B)$ be the regular representation as in (i) of Definition 1.1. Set

$$
{ }_{B} G^{c}=\left\{x \in G: \text { the function } g \hookrightarrow_{B} \pi\left(g x g^{-1}\right) \text { is norm continuous }\right\} .
$$

The following observation provides the technical tool which is the key to studying ${ }_{B} G^{c}$.

Theorem 1.4. Let $B,{ }_{B} \pi$ and ${ }_{B} G^{c}$ be as in Definition 1.3, and let $x \in G$. Then $x \in{ }_{B} G^{c}$ if and only if $C_{G}(x)$ is an open subgroup of $G$.

In view of Theorem 1.4 the subscript $B$ in ${ }_{B} G^{c}$ is redundant and will be omitted following the proof of this theorem. Furthermore, the superscript in $G^{c}$ may be read as referring to (norm) continuity or to (open) centralizers.

Proof. Suppose first that $C_{G}(x)$ is open in $G$. Then the function $g{\varkappa_{B}}_{B} \pi\left(g x g^{-1}\right)$ is constant when restricted to the open subgroup $C_{G}(x)$ of $G$, so in particular it is norm continuous at the identity. Since ${ }_{B} \pi$ is a norm-bounded representation of $G$ (1.1(i)), it follows that the function in question is continuous on all of $G$.

Conversely, suppose that $C_{G}(x)$ is not an open subgroup of $G$. Then any neighborhood $V$ of $e$ must contain some $v \in V \backslash C_{G}(x)$. We shall show that $\left\|_{B} \pi\left(g x g^{-1}\right)-{ }_{B} \pi(x)\right\|$ is bounded away from zero for all such $g$, hence $x \notin{ }_{B} G^{c}$.

As $[g, x] \neq e$ there is a neighborhood $W$ of $e$ such that $W \cap W[g, x]=\varnothing$. Let $0 \neq \varphi \in B$ such that $\varphi=0$ off $W$ and $\lambda(\varphi) \geqslant \delta\|\varphi\|$. Then ${ }_{B} \pi([g, x])$ is supported on $W[g, x]$. Thus

$$
\begin{aligned}
\left\|_{B} \pi\left(g x g^{-1}\right)-{ }_{B} \pi(x)\right\| & =\left\|\left({ }_{B} \pi([g, x])-I\right)_{B} \pi(x)\right\|>\left\|_{B} \pi([g, x])-I\right\| / C \\
& \geqslant\left\|_{B} \pi([g, x]) \varphi-\varphi\right\| / C\|\varphi\| \geqslant \lambda(\varphi) / C\|\varphi\| \geqslant \delta / C .
\end{aligned}
$$

COROLlaRY 1.5. Let $G$ be a connected group. Then $G^{c}=Z(G)$.

Proof. For any group $G$ one has $Z(G) \subset G^{c}$ trivially. Conversely, suppose that $x \in G^{c}$. Then $C_{G}(x)$ is an open subgroup of $G$. But $G$ is connected, so $C_{G}(x)=G$. Thus $x \in Z(G)$.

Corollary 1.6. Suppose that $G^{c}=G$. Then $G_{0} \subset Z(G)$.

The remainder of this note is devoted to the study of $G^{c}$. In $\$ 2$ we consider the case when $G=G^{c}$ and we obtain complete information when $G$ is compactly generated. $\$ 3$ is concerned with other cases. 
2. In this section we characterize the compactly generated groups for which $G=G^{c}$ and we obtain various equivalent formulations of this property.

Lemma 2.1. Suppose that $G=G^{c}$. Let $A$ and $B$ be compact sets of $G$. Then

$$
[A, B] \equiv\{[x, y]: x \in A, y \in B\}
$$

is a finite set.

Proof. Let $w, x, y, z \in G$ with

$$
z \in C_{G}(x) \cap C_{G}(y)
$$

and

$$
w \in C_{G}(x) \cap C_{G}(y) \cap C_{G}(z)
$$

Then

$$
\begin{aligned}
{[z x, w y] } & =z x w y x^{-1} z^{-1} y^{-1} w^{-1} \\
& =x y z w z^{-1} w^{-1} x^{-1} y^{-1} \quad \text { by (2.2) and (2.3) } \\
& =x y[z, w] x^{-1} y^{-1} \\
& =[x, y] \text { by (2.3). }
\end{aligned}
$$

Choose $x \in A$ and $y \in B$. Then there is a neighborhood $U_{x} \times V_{y}$ of $(x, y) \in$ $A \times B$ such that $[u, v]=[x, y]$ for all $(u, v) \in U_{x} \times V_{y}$. Pick a finite subcover from the open cover $\left\{U_{x} \times V_{y}\right\}$, and the lemma follows.

TheORem 2.4. Let $G$ be a compactly generated group. Then $G=G^{c}$ if and only if $Z(G)$ is an open subgroup of $G$.

Proof. As previously noted, $Z(G) \subset C_{G}(x)$ for all $x \in G$. If $Z(G)$ is open, then every group $C_{G}(x)$ is open, and hence $G^{c}=G$.

Conversely, suppose that $G=G^{c}$. Let $K$ be a compact neighborhood of $e$ which topologically generates $G$. It suffices to prove that $C_{G}(K)$ is open, since $C_{G}(K)=$ $Z(G)$. By Lemma 2.1, there is a neighborhood $U$ of $e$ with $U \subset K$ and $[K, K] \cap U$ $=\{e\}$. Let $V$ be a symmetric neighborhood of $e$ with $[V, V] \subset U$, so that $[V, V]=\{e\}$. Let $H$ be the subgroup of $G$ generated by $V$. Then $H$ is an abelian subgroup of $G$, and $H$ is open since $V$ is open. Choose $k_{1}, \ldots, k_{n} \in K$ such that $K \subset \cup \cup_{j=1}^{n} k_{j} H$. Let $L$ be the open subgroup of $G$ defined by

$$
L=H \cap \bigcap_{j=1}^{n} C_{G}\left(k_{j}\right) .
$$

If $k \in K$ then $k=k_{j} y$ for some $j$ and for some $y \in L$. Thus for any $x \in L$, one has $[k, x]=\left[k_{j} y, x\right]=e$, since $L \subset C_{G}\left(k_{j}\right)$. This shows that $L \subset C_{G}(K)$ and so $C_{G}(K)$ is an open subgroup, completing the proof.

EXAMPLE 2.5. The hypothesis that $G$ be compactly generated in Theorem 2.4 seems essential. We shall exhibit a group $G$ such that $G^{c}=G$ but $Z(G)$ is not open. Let $H_{0}$ be a finite abelian group, and set $H=\prod_{n=1}^{\infty} H_{n}$, where each $H_{n}$ is isomorphic to $H_{0}$. Let

$$
\Sigma=\bigoplus_{n=1}^{\infty}(Z(2))_{n},
$$


and let $\alpha: \Sigma \rightarrow \operatorname{Aut}(H)$ be the isomorphism such that the image of the generator of the $j$ th summand of $\Sigma$ interchanges the $2 j$ th and the $(2 j+1)$ th coordinates of elements of $H$. Set $G=H \times_{\alpha} \Sigma$ (semidirect product), where $\Sigma$ is given the discrete topology. Then $G$ is a locally compact group with open subgroup $H$. If $(h, \sigma) \in$ $C_{G}(x)$ for some element $x$ of $G$, then a direct computation shows that there exists a finite set $J$ of positive integers depending on $\sigma$ such that if $h_{k}^{\prime}=h_{k}$ for $k \in J$, then $\left(h^{\prime}, \sigma\right) \in C_{G}(x)$. Thus $C_{G}(x)$ is open in $G$. Hence $G^{c}=G$. On the other hand, $Z(G)$ consists only of elements whose $H$-coordinates are periodic with period two, so $Z(G)$ is not open in $G$.

THEOREM 2.6. The following conditions are equivalent for a locally compact group G.

(a) $G=G^{c}$.

(b) $C_{G}(x)$ is an open subgroup for all $x \in G$.

(c) $C_{G}(K)$ is open for all compact subsets $K$ of $G$.

(d) $C_{G}(H)$ is open for every compactly generated closed subgroup $H$ of $G$.

(e) $Z(H)$ is open in $H$ for every compactly generated closed subgroup $H$ of $G$.

Proof. The implications (a) $\Leftrightarrow(\mathrm{b})$ are the content of Theorem 1.4. The implication (b) $\Rightarrow$ (c) follows from the proof of Theorem 2.4. The facts that (b) $\Leftarrow$ (c) $\Leftrightarrow$ (d) $\Rightarrow(e)$ are routine. To see that (e) $\Rightarrow(d)$, recall that if $H$ is a compactly generated closed subgroup of $G$, then there is an open compactly generated subgroup $H^{\prime}$ of $G$ containing $H$. Applying (e) to $H^{\prime}$, we conclude that $C_{G}(H)$ is open in $G$.

THEOREM 2.7. Let $G$ be a compact group. Then the following conditions are equivalent.

(a) $G=G^{c}$.

(b) $G$ has finite conjugacy classes.

(c) $G$ is a central extension of an open abelian subgroup of finite index.

Proof. The implication (a) $\Leftrightarrow(b)$ is immediate from Lemma 2.1. If (a) holds, then $Z(G)$ is an open normal subgroup by Theorem 2.4 . Since $G$ is compact, any open subgroup must be of finite index, so (a) $\Rightarrow(c)$. (Note that the subgroup may be taken to be $Z(G)$.) Conversely, suppose that $H$ is an abelian subgroup of finite index in $G$ with $H$ central. Then $Z(G)$ is open, so $C_{G}(x)$ is open for all $x \in G$.

Condition (c) in Theorem 2.7 is parallel to a condition appearing in an important theorem of C. C. Moore, which we recall. A group $G$ is said to be of bounded degree if the dimensions of the irreducible unitary representations of $G$ are bounded [4]. The theorem of Moore [6] is as follows. The locally compact group $G$ is of bounded degree if and only if $G$ is an extension of an open abelian subgroup of finite index.

We see immediately that any compact group $G$ with $G=G^{c}$ must be of bounded degree, but that the converse is false. The simplest example was pointed out to us by I. Kaplansky: Take $G$ to be the noncentral extension of the circle group $T$ by the group of order two. Then $G$ is of bounded degree (in fact the irreducible unitary representations of $G$ have dimension at most two), but for appropriate choice $x, C_{G}(x)$ has four elements. So $G \neq G^{c}$; in fact $G^{c}=T$. 
3. $\$ 3$ is devoted to an exploration of how various conditions imposed upon $G^{c}$ are reflected in the structure of $G$.

THEOREM 3.1. $G^{c}$ is an open subgroup of $G$ if and only if $G$ has an open abelian subgroup.

Proof. If $H$ is an open abelian subgroup of $G$, then $H \subset C_{G}(x)$ for every $x \in H$. Hence $H \subset G^{c}$, so $G^{c}$ is open. Conversely, suppose that $G^{c}$ is open. Then $G^{c}$ contains an open compactly generated subgroup $H$. Theorem 2.4 implies that $Z(H)$ is open in $H$ and hence in $G$. Thus $Z(H)$ is an open abelian subgroup of $G$.

It would be of interest to characterize those groups $G$ with $G^{c}=\{e\}$. One motivation for our interest in this question is expressed by the following theorem (3.2). Of course, if $G$ is connected, then $G^{c}=\{e\}$ just means that $G$ has trivial center. A class of totally disconnected groups with $G^{c}=\{e\}$ is described in Example 3.3.

TheOREM 3.2. Let $\pi: G \rightarrow \mathscr{L}\left(L^{2}(G)\right)$ be the right regular representation, and for $x \in G$ let $\Phi_{x}$ be the automorphism of $\mathcal{L}_{G}$ given by

$$
\Phi_{x}(T)=\pi(x) T \pi(x)^{*} .
$$

If $x \in G \backslash G^{c}$, then $\Phi_{x}$ is outer. In particular, if $G^{c}=\{e\}$, then

$$
G \stackrel{\Phi}{\rightarrow} \operatorname{Aut}\left(\mathfrak{\varrho}_{G}\right) / \operatorname{Inn}\left(\mathfrak{E}_{G}\right)
$$

is injective.

Proof. For $x \in G \backslash G^{c}$ restrict $\Phi_{x}$ to the compact operators $\mathcal{K}$. Then $\Phi_{x}$ is implemented by conjugation by $\pi(x)$, and $\pi(x) \notin \mathcal{E}_{G}$. In fact, $\pi(x)$ is the only unitary operator implementing $\Phi_{x}$. For if $U$ is a unitary operator implementing $\Phi_{x}$, then $\pi(x) U^{-1}$ would centralize $\mathcal{K}$; hence $\pi(x)=U$. Thus there is no unitary element of $\mathscr{L}_{G}$ which implements $\Phi_{x}$, so $\Phi_{x}$ is outer.

ExAmple 3.3. Consider the group $\operatorname{SL}\left(2, Q_{p}\right)$, where $Q_{p}$ denotes the $p$-adic number field. Suppose that $x$ is an element of this group with an open centralizer. Then $x$ must commute with all matrices of the form $\left(\begin{array}{ll}1 & \alpha \\ \beta & 1\end{array}\right)$ for $\alpha, \beta$ sufficiently close to zero with $\alpha \beta=0$, since every neighborhood of $I$ contains such matrices. An easy computation shows that $x= \pm I$, so $\operatorname{SL}\left(2, Q_{p}\right)^{c}=Z(2)$. A similar computation yields $\operatorname{PSL}\left(2, Q_{p}\right)^{c}=\{e\}$.

More generally, let $G$ be a Zariski-connected semisimple affine algebraic group defined over a local field $k$ (of arbitrary characteristic), and assume that $G$ is almost simple and isotropic over $k$. Let $G(k)$ denote the group of $k$-rational points of $G$. Then $G(k)$ has a natural locally compact topology. Let $H$ be a closed cocompact subgroup of $G(k)$. Then every neighborhood of the identity in $H$ is dense in $G$ with respect to the Zariski topology [7, Lemma 2.1]. If $C_{H}(x)$ is open for some $x \in H$, then $x \in Z(G)$. Thus $H^{c}=Z(H)$. In particular, if $G$ has trivial center (e.g., $G$ is of adjoint type), then $H^{c}=\{e\}$.

In all of the examples considered so far in this note, $G^{c}$ is a closed subgroup of $G$. In Example 3.4 we show that this is not always the case. Although we do not 
know how to characterize those groups $G$ for which $G^{c}$ is closed, Theorem 3.5 below provides a step in that direction.

EXAMPLE 3.4. Let $H$ be a finite group with trivial center, and let $G=\prod_{n=1}^{\infty} H_{n}, H_{n}$ being isomorphic to $H$. Then it is easy to see that

$$
G^{c}=\left\{x=\left(x_{n}\right) \in G: x_{n}=e \text { for all but finitely many } n\right\}=\bigoplus_{1}^{\infty} H_{n} .
$$

In particular, $G^{c}$ is not closed in $G$.

TheOREM 3.5. Let $Y(G)=\bigcap_{x \in G^{c}} C_{G}(x)$.

(1) If $Y(G)$ is open in $G$, then $G^{c}$ is closed.

(2) If $G$ is metrizable and $G^{c}$ is compact, then $Y(G)$ is open.

Proof. The first assertion is clear. To prove the second assertion, let $\left\{H_{n}\right\}$ be a decreasing sequence of open subgroups of $G$ such that $G_{0}=\cap_{n=1}^{\infty} H_{n}$ and $H_{n} / G_{0}$ is compact for all $n$. Then every open subgroup of $G$ contains some $H_{n}$. For each $n$ let $G_{n}=\left\{x \in G^{c}: C_{G}(x) \supset H_{n}\right\}$. Then $\left\{G_{n}\right\}$ is an increasing sequence of closed subgroups of $G$ whose union is $G^{c}$. If $G^{c}$ is compact, then the Baire Category Theorem implies that $G_{k}$ is open in $G^{c}$ for some $k$, and hence $G_{m}=G^{c}$ for some $m \geqslant k$. Thus $H_{m} \subset Y(G)$.

Corollary 3.6. If $G_{0}$ is open in $G$, then $G^{c}$ is closed.

\section{REFERENCES}

1. P. Eymard, L'algèbre de Fourier d'un groupe localement compact, Bull. Math. Soc. France 92 (1964), 181-236.

2. A. Figà-Talamanca, Translation invariant operators in $L^{p}$, Duke Math. J. 32 (1965), 495-501.

3. A. Figà-Talamanca and G. I. Gaudry, Density and representation theorems for multipliers of type $(p, q)$, J. Austral. Math. Soc. 7 (1967), 1-6.

4. I. Kaplansky, Groups with representations of bounded degree, Canad. J. Math. 1 (1949), 105-112.

5. R. Loebl and C. Schochet, Covariant representations on the Calkin algebra. I, Duke Math. J. 45 (1978), 721-734.

6. C. C. Moore, Groups with finite dimensional irreducible representations, Trans. Amer. Math. Soc. 166 (1972), 401-410.

7. G. Prasad, Strong approximation for semi-simple groups over function fields, Ann. of Math. (2) 105 (1977), 553-572.

8. C. Schochet, Covariant representations on the Calkin algebra. II, Duke Math. J. 47 (1980), 451-460.

Department of Mathematics, Wayne State University, Detrort, Michigan 48202 